\title{
Amelocerebrohypohidrotic syndrome
}

INSERM

\section{Source}

INSERM. (1999). Orphanet: an online rare disease and orphan drug data base.

Amelocerebrohypohidrotic syndrome. ORPHA:1946

Kohlschütter-Tönz syndrome (KTS) is a genetically heterogeneous autosomal recessive syndrome characterized by the triad of amelogenesis imperfect, infantile onset epilepsy, intellectual disability with or without regression and dementia. 